Universidade de São Paulo

Escola Superior de Agricultura "Luiz de Queiroz"

Atributos do solo como indicadores de qualidade para avaliação de áreas em processo de restauração ativa na Mata Atlântica

\title{
Tatiana Cabral de Vasconcelos
}

Tese apresentada para obtenção do título de Doutora em Ciências, Programa: Recursos Florestais. Opção em:

Conservação de Ecossistemas Florestais

Piracicaba

2020 
Tatiana Cabral de Vasconcelos

Licenciada em Ciências Biológicas

Atributos do solo como indicadores de qualidade para avaliação de áreas em processo de restauração ativa na Mata Atlântica

Orientador:

Prof. Dr. RICARDO RIBEIRO RODRIGUES

Tese apresentada para obtenção do título de Doutora em Ciências, Programa: Recursos Florestais. Opção em: Conservação de Ecossistemas Florestais 


\section{Versão Simplificada}

\section{Resumo Geral}

\section{Atributos do solo como indicadores de qualidade para avaliação de áreas em processo de restauração ativa na Mata Atlântica}

No monitoramento da restauração florestal há uma diversidade de indicadores, porém, maior atenção é dispensada na avaliação do componente arbóreo, em detrimento à avaliação do componente edáfico. Neste trabalho avaliamos a qualidade do solo em diferentes tipos de uso, comumente encontrados em uma propriedade rural. O objetivo foi, aplicar um indicador de qualidade do solo que avalia os serviços do ecossistema (s.e.) do solo, por meio de um conjunto de 4 ou 6 indicadores combinados em um único Indicador Sintético de Qualidade do Solo (ISQS). A coleta de amostras de solo foi realizada no bioma Mata Atlântica, nas áreas das Fazendas Capoava, Jequitibá e Ingazinho, no município de Itu - SP. O cálculo dos indicadores foi realizado em sequências de análises multivariadas, primeiramente integramos 46 variáveis edáficas formando 04 conjuntos de dados: 16 variáveis químicas para o indicador de fertilidade do solo (FER), 09 variáveis físicas para o indicador de estrutura do solo (EST), 13 variáveis de macrofauna do solo para o indicador de diversidade do solo, 08 variáveis de agregação do solo para o indicador de agregação do solo (AGR). Testamos as hipóteses (i) a restauração florestal ativa (RA) com 5 anos de implantação tem maior qualidade do solo quando comparado com áreas de pastagem em uso contínuo (PUC) e pastagens abandonadas sem ações de restauração (PA); (ii) o solo de um fragmento de Floresta Estacional Semidecidual (FES) tem maior qualidade que os tipos de uso do solo (RA), (PUC) e (PA). Os resultados mostraram maior valor do ISQS na área FES como referência de conservação do ecossistema florestal. Posteriormente calculamos o indicador ciclagem de nutrientes (NUT), com 06 variáveis microbiológicas e o indicador de diversidade da mesofauna (DME), com 19 variáveis dos grupos taxonômicos da mesofauna na serapilheira. Os resultados mostraram que a restauração ativa após 5 anos de implantação apresentou média qualidade do solo, tanto com o cálculo do ISQS com 04 indicadores ou 06 indicadores. Apesar do indicador sintético de qualidade do solo, unir todas as váriaveis estudadas, a observação dos indicadores e suas relações também foi avaliada. Em nossos resultados foi explícita a variação conjunta dos indicadores de Estrutura do Solo (EST) com os indicadores de DMA e DME, que representam a macro e mesofauna do solo.

Palavras-chave: Restauração florestal, Restauração ativa, Indicador de qualidade do solo, Atributos do solo 


\section{ABSTRACT \\ Soil attributes as quality indicators for evaluating areas in the process of active restoration in the Atlantic Forest}

In the monitoring of forest restoration there is a diversity of indicators, however, more attention is given to the evaluation of the tree component, to the detriment of the evaluation of the edaphic component. In this work we evaluate the quality of the soil in different types of use, commonly found in a rural property. The objective was to apply a soil quality indicator that assesses soil ecosystem services (s.e.), using a set of 4 or 6 indicators combined into a single Synthetic Soil Quality Indicator (ISQS). The collection of soil samples was carried out in the Atlantic Forest biome, in the areas of the Capoava, Jequitibá and Ingazinho farms, in the municipality of Itu - SP. The calculation of the indicators was performed in sequences of multivariate analyzes, first we integrated 46 edaphic variables forming 04 data sets: 16 chemical variables for the soil fertility indicator (FER), 09 physical variables for the soil structure indicator (EST) , 13 soil macrofauna variables for the soil diversity indicator, 08 soil aggregation variables for the soil aggregation indicator (AGR). We tested the hypotheses (i) active forest restoration (RA) with 5 years of implantation has better soil quality when compared to pasture areas in continuous use (PUC) and abandoned pastures without restoration actions (PA); (ii) the soil of a fragment of Seasonal Semideciduous Forest (FES) has higher quality than the types of land use (RA), (PUC) and (PA). The results showed a greater value of the ISQS in the FES area as a reference for the conservation of the forest ecosystem. Subsequently, we calculated the nutrient cycling indicator (NUT), with 06 microbiological variables and the mesofauna diversity indicator (DME), with 19 variables from the mesofauna taxonomic groups in the litter. The results showed that the active restoration after 5 years of implantation showed average soil quality, both with the calculation of the ISQS with 04 indicators or 06 indicators. Despite the synthetic indicator of soil quality, uniting all the variables studied, the observation of the indicators and their relationships was also evaluated. In our results, the joint variation of the Soil Structure (EST) indicators with the DMA and DME indicators, which represent the soil macro and mesofauna, was explicit.

Keywords: Forest restoration, Active restoration, Soil quality indicator, Soil attributes 


\section{INTRODUÇÃO GERAL}

A Avaliação Ecossistêmica do Milênio (Millennium Ecosystem Assessment) (MEA, 2005), reconhece quatro categorias de benefícios às pessoas gerados pela natureza, ou seja, serviços ecossistêmicos (s.e.): serviços de provisão, serviços de regulação, serviços de suporte e serviços culturais, três deles estão relacionados diretamente com os processos que ocorrem no solo, tais como a ciclagem de nutrientes e formação do solo (Tabela 01). Dentre as principais potencialidades dos serviços ecossistêmicos provenientes do solo, destaca-se na restauração florestal a relação dos organismos do solo com a sucessão primária e secundária das florestas (VAN DER PUTTEN et al., 2009), a autoorganização molda mudança a longo prazo dos ecosistemas, onde o processo de sucessão ecológica depende da resiliência dos processos ecológicos mediados pela diversidade funcional dos organismos (HOLLING, 2001). Acreditamos que o incremento de vegetação nativa resultante do processo de restauração ecológica, pode permitir o aumento da diversidade da fauna do solo, que analisada de forma integrada com outros dados edáficos pode ser importante para aferir o retorno dos serviços ecossistêmicos que garantem a perpetuação da floresta, tornando-se mais uma ferramenta para o monitoramento da restauração florestal.

Tabela 01: Tipos de Serviços Ecossistêmicos relacionados aos invertebrados do solo. Fonte: (LAVELLE et al., 2006).

\begin{tabular}{|c|c|c|c|}
\hline $\begin{array}{l}\text { Tipo de Serviço } \\
\text { Ecossistêmico }\end{array}$ & Bem ou Serviço & Processo do Ecossistema & Contribuição dos Invertebrados do solo \\
\hline Provisão & $\begin{array}{l}\text { Suprimento de } \\
\text { água }\end{array}$ & $\begin{array}{c}\text { Infiltração e estoque de água nos poros do } \\
\text { solo. }\end{array}$ & $\begin{array}{c}\text { Construção e manutenção de porosidade estável } \\
\text { por meio da bioturbação e escavação. }\end{array}$ \\
\hline \multirow{3}{*}{ Suporte } & $\begin{array}{l}\text { Ciclagem de } \\
\text { Nutrientes }\end{array}$ & $\begin{array}{c}\text { Decomposição, Humificação, Regulação de } \\
\text { perdas de nutrientes (lixiviação e } \\
\text { denitrificação). }\end{array}$ & $\begin{array}{l}\text { Fragmentação dos resíduos vegetais, seleção e } \\
\text { ativação da atividade microbiana. }\end{array}$ \\
\hline & Formação do Solo & Pedogênese & $\begin{array}{c}\text { Bioturbação; deposição superficial, seleção de } \\
\text { partículas. }\end{array}$ \\
\hline & Produção Primária & $\begin{array}{l}\text { Estímulo de atividade simbiótica no solo; } \\
\text { produção indireta no solo de moléculas } \\
\text { reconhecidas pelas plantas como } \\
\text { hormônios; proteção contra pestes e } \\
\text { doenças. }\end{array}$ & $\begin{array}{c}\text { Aumento seletivo dos microrganismos e da } \\
\text { dominância funcional; Controle de pestes através } \\
\text { da interação biológica; aumento da capacidade } \\
\text { de resposta da planta. }\end{array}$ \\
\hline \multirow{4}{*}{ Regulação } & $\begin{array}{c}\text { Controle de } \\
\text { inundação e de }\end{array}$ & Regulação do escoamento da água. & $\begin{array}{c}\text { Criação de superfície rugosa devido as estruturas } \\
\text { biogênicas. }\end{array}$ \\
\hline & erosão & $\begin{array}{l}\text { Infiltração e armazenamento de água no } \\
\text { solo. }\end{array}$ & $\begin{array}{c}\text { Construção e manutenção de porosidade estável } \\
\text { por meio da bioturbação e escavação. }\end{array}$ \\
\hline & \multirow{2}{*}{$\begin{array}{l}\text { Regulação do } \\
\text { Clima }\end{array}$} & $\begin{array}{c}\text { Produção e consumo de gases do efeito } \\
\text { estufa. }\end{array}$ & $\begin{array}{l}\text { Sequestro de matéria orgânica na } \\
\text { macroagregados biogênicos. }\end{array}$ \\
\hline & & $\begin{array}{c}\text { Estoque de matéria orgânica no solo e na } \\
\text { biomassa. }\end{array}$ & $\begin{array}{l}\text { Aumento da formação de compostos húmicos } \\
\text { resistentes. }\end{array}$ \\
\hline
\end{tabular}

O avanço do conhecimento científico em restauração florestal demonstrou que o desenvolvimento dos ecossistemas é um processo dinâmico e afetado por múltiplos fatores, com destaque para distúrbios naturais e antrópicos que podem alterar a trajetória sucessional do ecossistema em recuperação ou até definir que essa trajetória permaneça estagnada ou em processo de regressão (BRANCALION; GANDOLFI; RODRIGUES, 2015) para estes autores é clara a necessidade de aprimoramento e revisão constante das metodologias, permitindo novas formas de enxergar e conduzir a restauração florestal.Somente com uma criteriosa avaliação do processo de restauração florestal é possível detectar falhas e propor ações corretivas, o monitoramento deve identificar se a trajetória atual está realmente levando a uma condição de ecossistema restaurado. Para a indicação do sucesso da restauração ecológica, na literatura predominam em $81 \%$ a $79 \%$ dos 
trabalhos a análise da vegetação, somente $6 \%$ utilizam fungos como indicadores, 5\% pássaros, 4\% invertebrados e 3\% mamíferos (GUERRA et al., 2020). Apenas 20\% dos estudos analisados por Valani et al. (2020) integravam os componentes físicos, químicos e biológicos para aferir a qualidade do solo, em tipos de uso solo e manejo analisados por estes autores, a maioria das publicaçõs usaram indicadores físicos $(80 \%)$, seguido pelos indicadores químicos $(70 \%)$ e com pouca atenção aos indicadores biológicos (33\%).

Dados sobre as condições do solo, durante o processo de restauração ecológica não estão incluídos nos indicadores convencionais, menos de um terço das publicações recentes em restauração ecológica no Brasil mencionam o solo (GUERRA et al., 2020). Nos ecossistemas tropicais a produção cientifica em restauração ecológica também possui pouca informação sobre o solo (ROMANELLI et al., 2018). Na avaliação de áreas em processo de restauração ecológica, alguns dados sobre o solo são incluídos de forma "burocrática" sem apropriada consideração (MENDES et al., 2019), sendo necessário desenvolver ferramentas para analisar o solo de forma dinâmica durante o processo de transformação de uso do solo.

A construção do conhecimento ecológico do solo na restauração ecológica (florestal) deve ser incentivado, normalmente restauradores pressupõem que os processos autogênicos da floresta começarão uma vez que estejam restabelecidas a composição e a estrutura apropriadas das espécies arbóreas, mas nem sempre essa pressuposição é válida(CALLAHAM; RHOADES; HENEGHAN, 2008; HENEGHAN et al., 2008). Para avaliar o solo durante o processo de sucessção ecológica é importante conhecer o conceito de ecologia acima e abaixo do solo (Figura 1) que pressupõe efeitos de feedback, oriundos das interações dos organismos do solo com o ambiente abiótico, que ocasionam as alterações observadas nos ecossistemas terrestres (VAN DER PUTTEN et al., 2009).

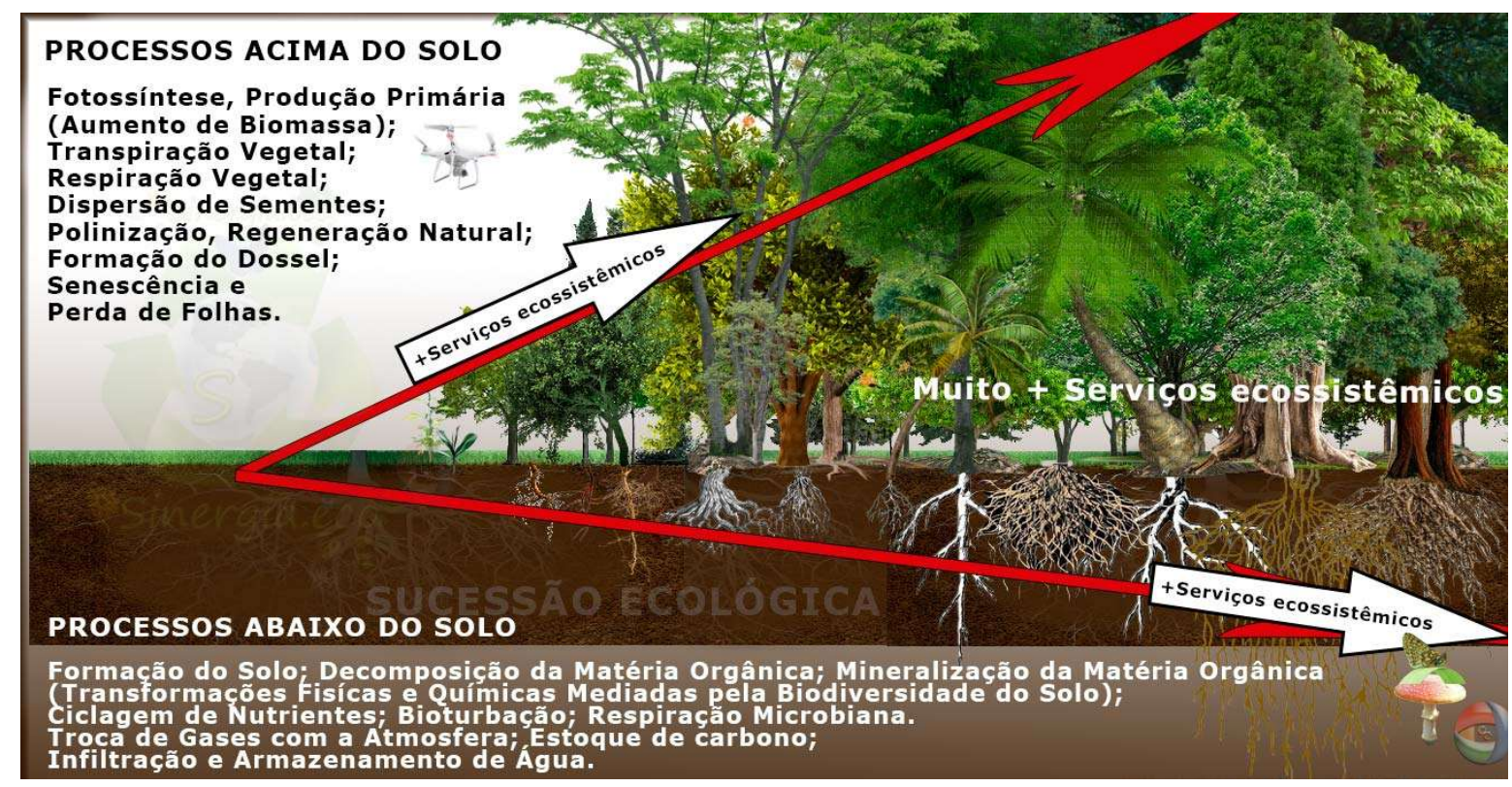

Figura 01: Ilustração da autora, solo e processos ecológicos que ocorrem na sucessão ecológica.

Em geral há uma diversidade de indicadores para monitoramento da restauração florestal ( LONDE et al., 2020; OLIVEIRA, 2011; RUIZ-JAEN \& AIDE, 2005), no entanto a melhor escolha de indicadores para verificar se a floresta foi recuperada, ainda estão em constante revisão (RODRIGUES et al., 2013; SUGANUMA; DURIGAN, 2015).

Dentre os indicadores recomendados para o monitoramento de áreas em processo de restauração florestal temos 3 categorias principais, com exemplos do indicador avaliado: 1.Composição: riqueza de espécie arbóreas; 2.Estrutura: 
porcentagem de cobertura do solo pelas copas das árvores e 3.Funcionamento: avaliação de um processo ecológico que irá garantir a autoperpetuação do ecossistema (VIANI et al., 2017; BRANCALION; GANDOLFI; RODRIGUES, 2015).

Normativas do estado de São Paulo trazem os valores crítico, mínimo e adequado, por exemplo, uma área com 5 anos de implantação da restauração ecológica, na Floresta Atlântica Estacional, deve apresentar, no mínimo, a riqueza de 3 a 10 espécies arbóreas nativas e 30 a 80\% de cobertura do solo pela vegetação nativa (Decreto SMA 32/14; Portaria CBRN 01/2015).

Elaborado em conjunto pelos atores do Pacto pela Restauração da Mata Atlântica (https://www.pactomataatlantica.org.br/) no protocolo de monitoramento para programas e projetos de restauração florestal (RODRIGUES et al., 2013), são consideradas a Fase I e a Fase II para o monitoramento da restauração florestal.

A Fase I representa a estruturação inicial do dossel, finda-se quando alcançado 70\% de cobertura do solo pelas espécies nativas. Fase II representa a dinâmica da trajetória ecológica, ou seja, devem ser observados os processos ecológicos. De acordo com o protocolo, a verificação do critério edáfico deve ser realizada através da fertilidade química e textura do solo, compactação do solo e conservação do solo (Tabela 2).

Tabela 2: Indicadores e verificadores do critério edáfico, do princípio ecológico do protocolo de monitoramento da restauração florestal, modificado de (RODRIGUES et al., 2013).

\begin{tabular}{ll}
\hline Indicador (Fase 1) & Verificador \\
\hline Fertilidade química & Disponibilidade de nutrientes, teor de matéria orgânica, pH, \\
metais pesados (quando necessário) \\
Granulometria & Análise de textura do solo (percentual de areia, argila e silte). \\
Compactação do solo & Resistência a penetração do solo (uso de penetrômetro) \\
& Ausência de boas práticas agrícolas \\
& Presença de erosão laminar (avaliação visual) \\
Conservação do solo & Avaliação visual da presença de solo descoberto na entrelinha \\
& (preparo convencional com revolvimento do solo) e da ausência \\
& de cultivo em nível. \\
\hline
\end{tabular}

O estado edáfico pode representar grande influência na trajetória da sucessão ecológica durante o processo da restauração florestal, logo após o plantio, a planta depende exclusivamente do potencial nutritivo do solo (1-3 meses), num momento intermediário (5 anos) já existem processos ecológicos evidenciados e ao final ( +20 anos) os processos ecossistêmicos podem chegar a pleno funcionamento (Figura 2).

O protocolo recomenda o uso dos critérios edáficos para avaliação inicial da área e durante a Fase 1 do monitoramento, onde o indicador de compactação do solo é um dos principais filtros para o bom desenvolvimento das plantas, nos casos em que a compactação é diagnosticada, ações corretivas de descompactação com uso de implementos escarificadores, subsoladores podem ser recomendadas. Para avaliação dos parâmetros químicos como macronutrientes, pH, conteúdo de matéria orgânica, Capacidade de Troca Catiônica (CTC). Porém, não existem parâmetros reconhecidos para avaliar a restauração florestal, de acordo, com o protocolo podem ser utilizados os mesmos parâmetros da avaliação de solo agrícolas. No entanto, a ciclagem dos nutrientes em ambiente florestal é diferenciada dos sistemas agrícolas, onde o uso de fertilizantes é mais intenso que no plantio de espécies arbóreas (GONÇALVES, 2000).

O principal requisito para um solo de boa qualidade, é a presença de vida do solo. A biota do solo está interligada aos processos de formação do solo, manutenção da fertilidade e qualidade do solo, pois atua nas principais funções ecológicas do ecossistema terrestre (MOREIRA, 2008). Por exemplo, a macrofauna do solo pode atuar em diversos níveis tróficos da teia alimentar do solo (Figura 3), levando a utilização destes organismos como bioindicadores. 


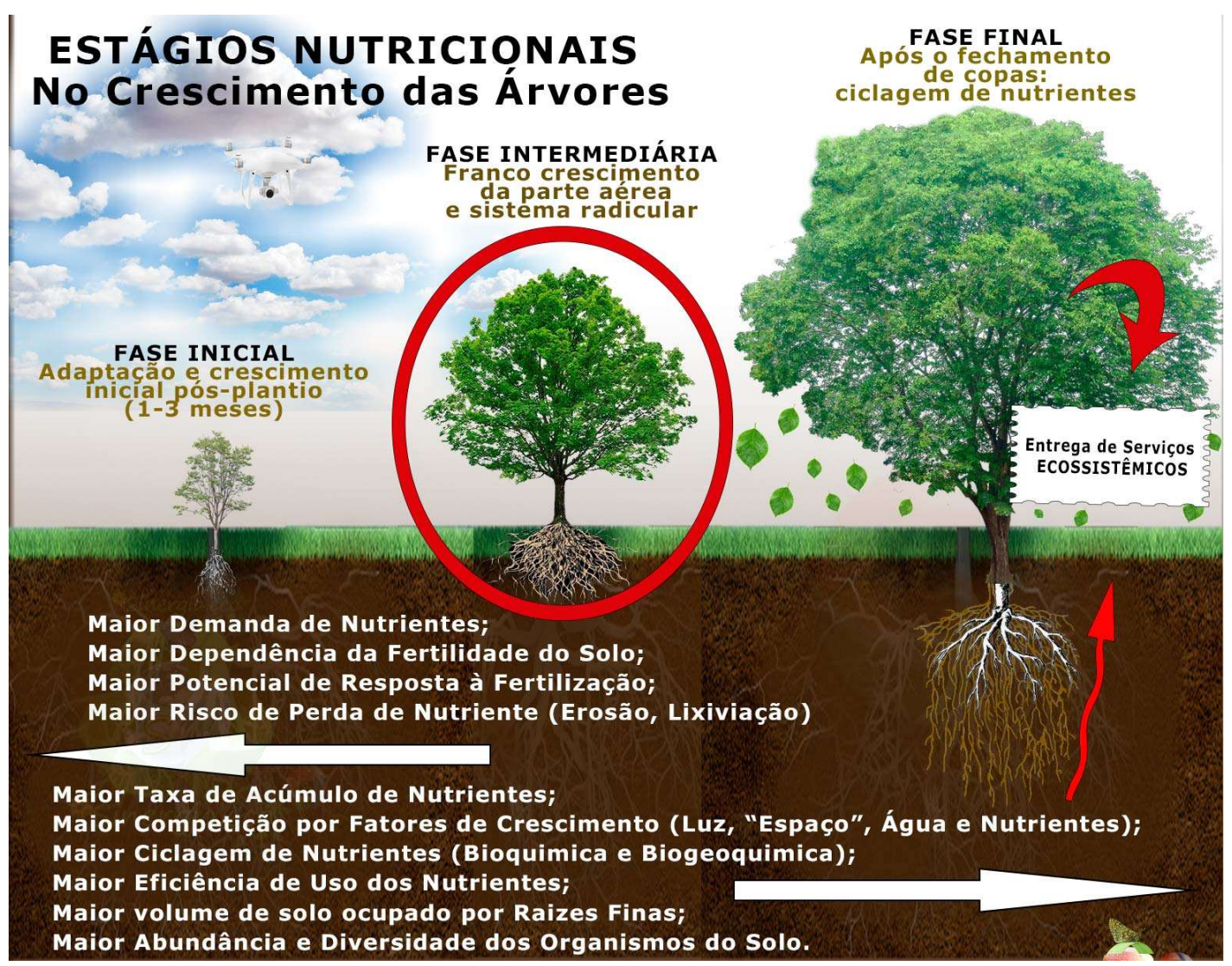

Figura 2: Fase inicial de plantio da espécie arbórea (1-3meses) ocorre adaptação e crescimento, Fase Intermediária (5 anos) ocorre franco crescimento da parte área e radicular, Fase Final (>20anos) ocorre o fechamento de copas e estabilidade na ciclagem de nutrientes, que resulta da maior provisão de Serviços Ecossistêmicos. Modificado de (GONÇALVES, 2000).

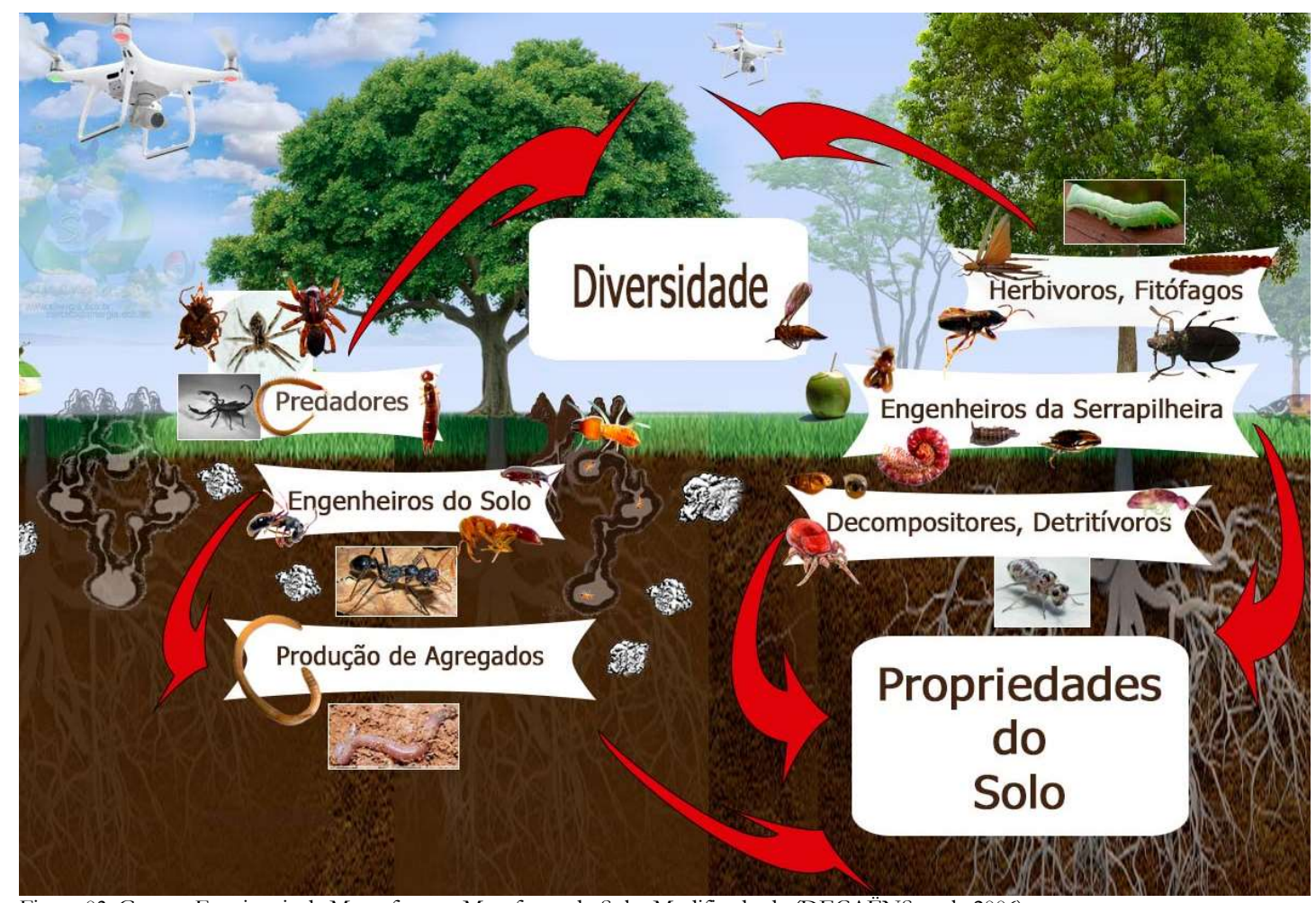

Figura 03: Grupos Funcionais da Macrofauna e Mesofauna do Solo. Modificado de (DECAËNS et al., 2006). 
Os serviços ecossistêmicos da biodiversidade do solo devem ser melhor compreendidos a fim de usar seu potencial para verificar a sustentabilidade dos ecossistemas terrestres (DOMINATI; PATTERSON; MACKAY, 2010). Diversos atributos do solo quando analisados separadamente podem caracterizar determinado processo na dinâmica do funcionamento do solo, porém são necessários valores comparáveis e indicadores replicáveis para possibilitar o monitoramento das áreas em processo de restauração ecológica.

Indicadores multifuncionais da qualidade do solo foram construídos com dados de biodiversidade do solo, propriedades físicas, propriedade químicas e agregação do solo, como o índice General Indicator of Soil Quality (GISQ, acrônimo em inglês), proposto por (VELASQUEZ; LAVELLE; ANDRADE, 2007). Esta abordagem compreende a avaliação dos diferentes serviços ecossistêmicos fornecidos pelo solo usando conjuntos relevantes de variáveis (VELASQUEZ; LAVELLE, 2019). Este índice é dinâmico e prático, as informações edáficas são apresentadas de forma sintética trazem à tona as diferenças entre os tipos de uso do solo (BÜNEMANN et al., 2018; DOMÍNGUEZ-HAYDAR et al., 2019; LAVELLE et al., 2014; MARICHAL et al., 2014; NURIA et al., 2011; VELASQUEZ; LAVELLE; ANDRADE, 2007).

Neste trabalho avaliamos a qualidade do solo, em 4 diferentes tipos de uso: restauração florestal ativa (RA) com 5 anos de implantação; pastagens abandonadas sem ações de restauração (PA); áreas de pastagem em uso contínuo (PUC) e um fragmento de Floresta Estacional Semidecidual (FES), situações ambientais comumente encontradas em uma propriedade rural. O objetivo foi aplicar um indicador de qualidade do solo que avalia os serviços do ecossistema (s.e.) do solo por meio de um conjunto de 4 ou 6 indicadores combinados em um único Indicador Sintético de Qualidade do Solo (ISQS). Integramos diversas variáveis edáficas, cujos indicadores representam um tipo de serviço ecossistêmico do solo: 16 variáveis químicas para o indicador de fertilidade do solo (FER), 09 variáveis físicas para o indicador de estrutura do solo (EST), 13 variáveis de macrofauna do solo para o indicador de diversidade do solo, 08 variáveis de agregação do solo para o indicador de agregação do solo (AGR), 06 variáveis microbiológicas para o indicador de ciclagem de nutrientes (NUT) e 19 variáveis dos grupos taxonômicos da mesofauna na serapilheira para o indicador de diversidade da mesofauna (DME). Testamos a diferença dos resultados de cada um dos indicadores entre os diferentes tipos de uso do solo e testamos o cálculo do ISQS com 4 e 6 indicadores.

\section{REFERÊNCIAS}

ARONSON, J. What can and should be legalized in ecological restoration? Revista Árvore, v. 34, n. 3, p. 451-454, 2010 .

BRANCALION, P. H. S.; GANDOLFI, S.; RODRIGUES, R. R. Restauração florestal. São Paulo: Oficina de Textos, 2015.

BÜNEMANN, E. K. et al. Soil quality - A critical review. Soil Biology and Biochemistry, v. 120, n. September 2017, p. 105-125, 2018.

CALLAHAM, M. A.; RHOADES, C. C.; HENEGHAN, L. A striking profile: Soil ecological knowledge in restoration management and science. Restoration Ecology, 2008.

DECAËNS, T. et al. The values of soil animals for conservation biology. European Journal of Soil Biology, v. 42, n. SUPPL. 1, 2006.

DOMINATI, E.; PATTERSON, M.; MACKAY, A. A framework for classifying and quantifying the natural capital and ecosystem services of soils. Ecological Economics, v. 69, n. 9, p. 1858-1868, 2010.

DOMÍNGUEZ-HAYDAR, Y. et al. Evaluation of reclamation success in an open-pit coal mine using integrated soil physical, chemical and biological quality indicators. Ecological Indicators, v. 103, n. March, p. 182-193, 2019. 
GONÇALVES, J. L. DE M. Nutrição e fertilização florestal. Piracicaba: Piracicaba FEALQ, 2000.

GUERRA, A. et al. Ecological restoration in Brazilian biomes: Identifying advances and gaps. Forest Ecology and Management, v. 458, n. December, 2020.

HENEGHAN, L. et al. Integrating Soil Ecological Knowledge into Restoration Management. Restoration Ecology, v. 16, n. 4, p. 608-617, dez. 2008.

HOLLING, C. S. Understanding the complexity of economic, ecological, and social systems. Ecosystems, v. 4, n. 5, p. 390-405, 2001.

LAVELLE, P. et al. Soil invertebrates and ecosystem services. European Journal of Soil Biology, v. 42, n. SUPPL. 1, nov. 2006.

LAVELLE, P. et al. Soil ecosystem services and land use in the rapidly changing orinoco river basin of colombia. Agriculture, Ecosystems and Environment, v. 185, p. 106-117, 2014.

LONDE, V. et al. Reference and comparison values for ecological indicators in assessing restoration areas in the Atlantic Forest. Ecological Indicators, v. 110, 1 mar. 2020.

MARICHAL, R. et al. Soil macroinvertebrate communities and ecosystem services in deforested landscapes of Amazonia. Applied Soil Ecology, v. 83, p. 177-185, 2014.

MEA. Millennium Ecosystem Assessment. Summary for decision makers. [s.l: s.n.].

MENDES, M. S. et al. Look down-there is a gap-the need to include soil data in Atlantic Forest restoration. Restoration Ecology, v. 27, n. 2, p. 361-370, 2019.

MOREIRA, F. M. S. Biodiversidade do solo em ecossistemas brasileiros. Lavras: Lavras UFLA, 2008.

NURIA, R. et al. IBQS: A synthetic index of soil quality based on soil macro-invertebrate communities. Soil Biology and Biochemistry, v. 43, n. 10, p. 2032-2045, 2011.

OLIVEIRA, R. O estado da arte da ecologia da restauração e sua relação com a restauração de ecossistemas florestais no bioma Mata Atlântica. Aleph, 2011.

RODRIgUeS, R. R. et al. Protocolo de Monitoramento para Programas e Projetos de Restauração FlorestalPacto pela Restauração Florestal da Mata Atlântica, 2013.

ROMANELLI, J. P. et al. Assessing ecological restoration as a research topic using bibliometric indicators. Ecological Engineering, v. 120, n. March, p. 311-320, 2018.

RUIZ-JAEN, M. C.; AIDE, T. M. Restoration success: How is it being measured? Restoration Ecology, v. 13, n. 3, p. 569-577, 2005.

SUGANUMA, M. S.; DURIGAN, G. Indicators of restoration success in riparian tropical forests using multiple reference ecosystems. Restoration Ecology, v. 23, n. 3, p. 238-251, 2015.

VELASQUEZ, E.; LAVELLE, P. Soil macrofauna as an indicator for evaluating soil based ecosystem services in agricultural landscapes. Acta Oecologica, v. 100, n. September, p. 103446, 2019.

VELASQUEZ, E.; LAVELLE, P.; ANDRADE, M. GISQ, a multifunctional indicator of soil quality. Soil Biology and Biochemistry, 2007.

VIANI, R. A. G. et al. Protocol for monitoring tropical forest restoration: Perspectives from the atlantic forest restoration pact in Brazil. Tropical Conservation Science, v. 10, 2017. 
Artigo 1: 


\title{
USO DO INDICADOR SINTÉTICO DE QUALIDADE DO SOLO PARA AVALIAÇÃO DE ÁREAS EM PROCESSO DE RESTAURAÇÃO FLORESTAL ATIVA COM 5 ANOS DE IMPLANTAÇÃO NA FLORESTA ATLÂNTICA, SÃO PAULO, BRASIL.
}

\author{
Tatiana Cabral de Vasconcelos ${ }^{1}$, Elena Velasquez ${ }^{2}$, Patrick Lavelle, Ricardo Ribeiro Rodrigues ${ }^{3}$ \\ 1.Escola Superior de Agricultura Luiz de Queiroz, Universidade de São Paulo, Piracicaba, SP, Brasil; 2. Universidad Nacional de Colombia, sede Palmira, \\ Valle do Cauca, Colômbia. 3.Líder do Laboratório de Ecologia e Restauração Ecológica, Escola Superior de Agricultura Luiz de Queiroz, Universidade de São Paulo, \\ Piracicaba, SP, Brasil.
}

\section{Resumo}

A restauração florestal é um processo dinâmico e afetado por múltiplos fatores. A trajetória sucessional do ecossistema em recuperação tem sido avaliada com indicadores de diversidade e estrutura da vegetação, enquanto os processos ecológicos do solo raramente são considerados. A análise do solo pode ser realizada medindo seus atributos físicos, químicos e biológicos de forma isolada ou combinada gerando indicadores compostos, os quais permitem observar os processos do ecossistema representando um indicador da categoria de funcionamento. O objetivo deste trabalho foi integrar 46 variáveis edáficas (16 variáveis químicas, 09 variáveis de estrutura do solo, 13 de diversidade da macrofauna e 08 de agregação do solo) que representam respectivamente os indicadores de: fertilidade do solo (FER), estrutura do solo (EST), diversidade da macrofauna (DMA) e agregação do solo (AGR) utilizados na composição do Índice Sintético de Qualidade do Solo (ISQS). A coleta de amostras foi realizada em 4 tipos de usos do solo, nas Fazendas Capoava, Jequitibá e Ingazinho (Itu, São Paulo, Brasil) em junho de 2017. Para a construção dos indicadores foram analisadas: amostras deformadas e indeformadas na profundidade de $0-10 \mathrm{~cm}$. Testamos as hipóteses: (i) a restauração ativa (RA) com 5 anos de implantação tem maior qualidade do solo quando comparado com áreas de pastagem em uso contínuo (PUC) e pastagens abandonadas sem ações de restauração (PA) e (ii) o solo de um fragmento de Floresta Estacional Semidecidual (FES) em estágio médio de conservação tem maior qualidade do solo quando comparada com a restauração ativa (RA), áreas de pastagem em uso contínuo (PUC) e pastagens abandonadas sem ações de restauração (PA). Os resultados descrevem um gradiente específico para cada indicador, em RA encontramos alta qualidade dos indicadores EST e DMA. Na área PA encontramos alta qualidade do indicador de AGR, em PUC encontramos baixa qualidade dos indicadores EST e DMA. O padrão dado pelo ISQS foi $\mathrm{FES}>\mathrm{RA}>\mathrm{PA}>\mathrm{PUC}$, o valor do ISQS $=0,59$ RA indicou média qualidade do solo apesar de mais alto não diferiu significativamente de PA, com ISQS=0,56 e de PUC com ISQS $=0,38$ refutando a primeira hipótese. $\mathrm{Na}$ área FES encontramos alta qualidade do solo com ISQS $=0,80$ diferindo significativamente de todos outros tipos de usos do solo, corroborando a segunda hipótese. O ISQS funcionou como ferramenta para transformar um número elevado de dados, em informações numéricas que permitiram distinguir a qualidade do solo sob diferentes tipos de uso. Concluímos que após 5 anos de implantação da restauração ativa na Mata Atlântica é possível obter uma qualidade média em relação ao componente edáfico observado de forma completa através do ISQS.

Palavras chaves: 1. Restauração ativa 2. Atributos do solo 3. Índice Sintético de Qualidade do Solo 4. Indicadores.

\begin{abstract}
Forest restoration is a dynamic process and affected by multiple factors. The successional trajectory of the recovering ecosystem has been evaluated with indicators of diversity and structure of vegetation, while ecological soil processes are rarely considered. Soil analysis can be performed by measuring its physical, chemical and biological attributes in an isolated or combined way, generating composite indicators, which allow observing the ecosystem processes representing an indicator of the functioning category. The objective of this work was to integrate 46 edaphic variables (16 chemical variables, 09 variables of soil structure, 13 of macrofauna diversity and 08 of soil aggregation) which respectively represent the indicators of: soil fertility (FER), soil structure (EST), macrofauna diversity (DMA) and soil aggregation (AGR) used in the composition of the Synthetic Index of Soil Quality (ISQS). Sample collection was carried out in 4 types of land use, at the Capoava, Jequitibá and Ingazinho Farms (Itu, São Paulo, Brazil) in June 2017. For the construction of the indicators, the following were analyzed: deformed and undisturbed samples at a depth of $0-10 \mathrm{~cm}$. We tested the hypotheses: (i) active restoration (RA) with 5 years of implantation has better soil quality when compared to pasture areas in continuous use (PUC) and abandoned pastures without restoration actions (PA) and (ii) the soil of a fragment of Seasonal Semideciduous Forest (FES) in medium conservation stage has higher soil quality when compared to active restoration (RA), pasture areas in continuous use (PUC) and abandoned pastures without restoration actions (PA). The results describe a specific gradient for each indicator, in RA we find high quality of the EST and DMA indicators. In the PA area we find high quality of the AGR indicator, in PUC we find low quality of the EST and DMA indicators. The standard given by ISQS was FES $>$ RA $>$ PA $>$ PUC, the value of ISQS $=0.59$
\end{abstract}


RA indicated average soil quality, although higher, it did not differ significantly from PA, with ISQS $=0.56$ and PUC with ISQS $=0.38$ refuting the first hypothesis. In the FES area, we found high soil quality with ISQS $=0.80$, differing significantly from all other types of land uses, corroborating the second hypothesis. ISQS functioned as a tool to transform a large number of data into numerical information that allowed to distinguish the quality of the soil under different types of use. We conclude that after 5 years of implementation of active restoration in the Atlantic Forest it is possible to obtain an average quality in relation to the edaphic component observed in a complete way through ISQS.

Key words: 1. Active restoration 2. Soil attributes 3. General Index Soil Quality 4. Indicators

\section{INTRODUÇÃO}

A restauração ecológica é uma das principais estratégias para aliar a conservação da natureza, com retorno da biodiversidade e dos serviços ecossistêmicos em áreas rurais (BRANCALION; GANDOLFI; RODRIGUES, 2015). Atualmente a demanda por ações de restauração dos ecossistemas é latente no Brasil e no mundo (STRASSBURG; IRIBARREM; BEYER, 2020).

Os projetos de restauração, após implantados devem ser devidamente acompanhados, o monitoramento é fundamental, para determinar o alcance das metas estabelecidas (VIANI et al., 2017). Dentre os indicadores recomendados para o monitoramento de áreas em processo de restauração florestal temos 3 categorias principais, com exemplos do indicador avaliado: 1.Composição: riqueza de espécie arbóreas; 2.Estrutura: porcentagem de cobertura do solo pelas copas das árvores e 3.Funcionamento: avaliação de um processo ecológico que irá garantir a autoperpetuação do ecossistema (VIANI et al., 2017; BRANCALION; GANDOLFI; RODRIGUES, 2015).

As medidas de processos ecológicos fazem parte da categoria de funcionamento e trabalhos que utilizaram estas métricas tem aumentando na literatura (WORTLEY; HERO; HOWES, 2013), adicionalmente a Floresta Tropical tem grande aplicabilidade para bioindicadores (GATICA-SAAVEDRA; ECHEVERRÍA; NELSON, 2017). Para capturar a variação existente nos ecossistemas é recomendada a avaliação de uma ou mais áreas de referência (RUIZ-JAEN; AIDE, 2005), para todos os biomas é preciso definir os valores de referência baseados em florestas maduras para comparar com os resultados do monitoramento (LONDE et al., 2020).

Para utilização dos processos ecológicos que ocorrem no solo, é necessário desenvolver ferramentas para analisar o solo de forma dinâmica, infelizmente, na avaliação de áreas em processo de restauração ecológica, alguns dados sobre o solo quando presentes, não recebem apropriada consideração (MENDES et al., 2019).

Greiner et al. (2017) apontam que a maioria dos estudos realizados na Europa, reportam poucas propriedades do solo e uma abordagem multifuncional do solo é necessária. O conceito de qualidade do solo representa, a capacidade de funcionamento do solo dentro das limitações do ecossistema local (DORAN, 2002). A qualidade do solo é um conceito funcional complexo, inferido por meio de indicadores obtidos das propriedades mensuráveis do solo (CHERUBIN et al., 2016).

O objetivo deste trabalho foi aplicar a metodologia desenvolvida por (VELASQUEZ; LAVELLE; ANDRADE, 2007) através do cálculo do General Indicator of Soil Quality (GISQ, acrônimo em inglês), aqui denominado Indicador Sintético de Qualidade do Solo (ISQS). Avaliamos a qualidade do solo em quatro tipos de uso no bioma Mata Atlântica: 1) restauração florestal ativa (RA) com 5 anos de implantação, 2)pastagens abandonadas sem ações de restauração (PA), 3)áreas de pastagem em uso contínuo (PUC) e 4) fragmento de Floresta Estacional Semidecidual (FES).

Utilizando análises multivariadas calculamos 4 indicadores: 16 variáveis químicas para o indicador de fertilidade do solo (FER), 09 variáveis físicas para o indicador de estrutura do solo (EST), 13 variáveis de macrofauna do solo para o 
indicador de diversidade do solo (DMA) e 08 variáveis de morfologia do solo para o indicador de agregação do solo (AGR). Integramos os 4 indicadores (FER+EST+DMA+AGR) em um único Indicador Sintético de Qualidade do Solo (ISQS) Testamos as hipóteses:

(i) A restauração florestal ativa (RA) com 5 anos de implantação tem maior qualidade do solo quando comparado com áreas de pastagem em uso contínuo (PUC) e pastagens abandonadas sem ações de restauração (PA).

(ii) $\mathrm{O}$ solo de um fragmento de Floresta Estacional Semidecidual (FES) em estágio médio de conservação tem maior qualidade do solo quando comparada com a restauração florestal ativa (RA), áreas de pastagem em uso contínuo (PUC) e pastagens abandonadas sem ações de restauração (PA).

\section{CONCLUSÃO}

O ISQS funcionou como ferramenta eficiente para transformar um número elevado de dados do solo em informações numéricas que permitiram distinguir quais áreas possuem alta, média ou baixa qualidade do solo. O ISQS foi sensível as alterações entre os tipos de uso do solo, a alta variabilidade indica o potencial de melhorias nas áreas com indicadores baixos. A referência positiva em termos de qualidade do solo foi o fragmento de floresta atlântica estacional semidecidual, permitindo ao restaurador determinar características do solo em um ambiente desejável e a referência negativa a pastagem de uso contínuo. Nas áreas em processo de restauração ativa com 5 anos, a qualidade do solo é maior do que na pastagem sem ações de restauração pelo mesmo período de tempo. O fragmento florestal, mesmo inserido numa paisagem bastante alterada antropicamente pode manter seus processos ecológicos em estabilidade e bom funcionamento. Concluímos que após 5 anos de implantação da restauração ativa na Mata Atlântica é possível obter uma qualidade média em relação ao componente edáfico observado de forma completa através do ISQS.

\section{REFERÊNCIAS}

AMAZONAS, N. T. et al. Soil macrofauna density and diversity across a chronosequence of tropical forest restoration in southeastern Brazil. Brazilian Journal of Biology, v. 78, n. 3, p. 449-456, 2018.

BRANCALION, P. H. S.; GANDOLFI, S.; RODRIGUES, R. R. Restauração florestal. São Paulo: Oficina de Textos, 2015.

DOMÍNGUEZ-HAYDAR, Y. et al. Evaluation of reclamation success in an open-pit coal mine using integrated soil physical, chemical and biological quality indicators. Ecological Indicators, v. 103, n. March, p. 182-193, 2019.

GATICA-SAAVEDRA, P.; ECHEVERRÍA, C.; NELSON, C. R. Ecological indicators for assessing ecological success of forest restoration : a world review. v. 25, n. 6, p. 850-857, 2017.

GREINER, L. et al. Soil function assessment: review of methods for quantifying the contributions of soils to ecosystem services. Land Use Policy, v. 69, n. September, p. 224-237, 2017.

LAVELLE, P. et al. Soil ecosystem services and land use in the rapidly changing orinoco river basin of colombia. Agriculture, Ecosystems and Environment, v. 185, p. 106-117, 2014.

LAVELLE, P. et al. Soil aggregation, ecosystem engineers and the C cycle. Acta Oecologica, v. 105, n. December 2019, p. 103561, 2020.

LONDE, V. et al. Reference and comparison values for ecological indicators in assessing restoration areas in the Atlantic Forest. Ecological Indicators, v. 110, 1 mar. 2020.

MARICHAL, R. et al. Soil macroinvertebrate communities and ecosystem services in deforested landscapes of 
Amazonia. Applied Soil Ecology, v. 83, p. 177-185, 2014.

MEA, Millennium Ecosystem Assessment series: Ecosystems and Human Well-being: General Synthesis. Island Press, 160pp, 2005.

MENDES, M. S. et al. Look down-there is a gap-the need to include soil data in Atlantic Forest restoration. Restoration Ecology, v. 27, n. 2, p. 361-370, 2019.

PEREIRA, N. A. et al. Does the Process of Passive Forest Restoration Affect the Hydrophysical Attributes of the Soil Superficial Horizon? n. 2011, p. 1-15, 2020.

RODRIGUES, R. R.; BRANCALION, P. H. S.; NAVE, A. PROGRAMA DE ADEQUAÇÃo AMBIENTAL E RESTAURAÇÃO ECOLÓGICA DAS FAZENDAS CAPOAVA , JEQUITIBÁ E INGAZINHO. Piracicaba, 2012.

ROSSI, M. Mapa pedológico do Estado de São Paulo: revisado e ampliado. São Paulo: [s.n.]. v. 124

RUIZ-JAEN, M. C.; AIDE, T. M. Restoration success: How is it being measured? Restoration Ecology, v. 13, n. 3, p. 569-577, 2005.

SNYDER, B. A.; HENDRIX, P. F. Current and potential roles of soil macroinvertebrates (earthworms, millipedes, and isopods) in ecological restoration. Restoration Ecology, v. 16, n. 4, p. 629-636, 2008.

STRASSBURG, B. B. N.; IRIBARREM, A.; BEYER, H. L. Global priority areas for ecosystem restoration. n. August 2019, 2020.

VASCONCELLOS, R. L. F. et al. Soil macrofauna as an indicator of soil quality in an undisturbed riparian forest and recovering sites of different ages. European Journal of Soil Biology, v. 58, p. 105-112, 2013.

VELASQUEZ, E. et al. This ped is my ped: Visual separation and near infrared spectra allow determination of the origins of soil macroaggregates. Pedobiologia, 2007.

VELASQUEZ, E.; LAVELLE, P.; ANDRADE, M. GISQ, a multifunctional indicator of soil quality. Soil Biology and Biochemistry, 2007.

VELOSO, H. P. Classificação da Vegetação Brasileira, Adaptada a um Sistema UniversalRio de Janeiro, 1991. VIANI, R. A. G. et al. Protocol for monitoring tropical forest restoration: Perspectives from the atlantic forest restoration pact in Brazil. Tropical Conservation Science, v. 10, 2017.

WORTLEY, L.; HERO, J. M.; HOWES, M. Evaluating ecological restoration success: A review of the literature. Restoration Ecology, v. 21, n. 5, p. 537-543, 2013. 
Artigo 2: 


\section{INTEGRAÇÃO DE ATRIBUTOS MICROBIOLÓGICOS E DIVERSIDADE DA MESOFAUNA DA SERAPILHEIRA NO CÁLCULO DO INDICADOR SINTÉTICO DE QUALIDADE DO SOLO - ISQS}

Tatiana Cabral de Vasconcelos ${ }^{1}$, Elena Velasquez ${ }^{2}$, Patrick Lavelle, Ricardo Ribeiro Rodrigues ${ }^{3}$

1.Escola Superior de Agricultura Luiz de Queiroz, Universidade de São Paulo, Piracicaba, SP, Brasil; 2. Universidad Nacional de Colombia, sede Palmira, Valle do Cauca, Colômbia. 3.Líder do Laboratório de Ecologia e Restauração Ecológica, Escola Superior de Agricultura Luiz de Queiroz, Universidade de São Paulo, Piracicaba, SP, Brasil.

\section{RESUMO}

$\mathrm{Na}$ restauração florestal é essencial restabelecer os processos ecológicos do solo, principalmente, nos projetos que possuem metas relacionadas com mitigação das mudanças climáticas, proteção do solo e conservação da água, neste caso, avaliar a biota do solo pode ser importante. Atributos microbiológicos do solo e invertebrados da mesofauna que vivem na serapilheira, podem trazer informações sobre o funcionamento do solo em diferentes tipos de uso e manejo do solo. Pontos convergentes entre os diversos índices de qualidade do solo consistem em escolher os mais relevantes atributos do solo, para integra-los em um valor único que varia em escala de qualidade alta, média ou baixa. O objetivo deste trabalho foi integrar os atributos microbiológicos e diversidade da mesofauna da serapilheira como indicadores adicionais no cálculo do General Indicator of Soil Quality (GISQ, acrônimo em inglês), aqui denominado Indicador Sintético de Qualidade do Solo (ISQS), metodologia desenvolvida por (VELASQUEZ; LAVELLE; ANDRADE, 2007). Utilizando análises multivariadas calculamos os indicadores: 1) Ciclagem de nutrientes (NUT): inclui 06 atributos microbiológicos do solo e 2) Diversidade da mesofauna na serapilheira (DME): inclui dados da densidade (ind $/ \mathrm{m}^{2}$ ) de 18 Grupos Taxonômicos (GT) da mesofauna na serapilheira e o valor da Riqueza ( $n^{\circ}$ de GT). Avaliamos a diferença destes indicadores em relação aos tipos de uso do solo: FES (Fragmento de Floresta Estacional Semidecidual); RAA (Restauração Ativa A); RAB (Restauração Ativa B); RAC (Restauração Ativa C); PUC (Pastagem de uso contínuo); PAA (Pastagem Abandonada A); PAB (Pastagem Abandonada B); PAC (Pastagem Abandonada C), no bioma Mata Atlântica, em Itu, São Paulo, Brasil, nas áreas das Fazendas Capoava, Jequitibá e Ingazinho. Testamos a hipótese de que o valor do índice sintético de qualidade do solo (ISQS), em áreas em processo de restauração ativa é alterado quando calculado: utilizando quatro indicadores (FER, EST, DMA, AGR) ou seis indicadores (NUT, DME, FER, EST, DMA, AGR). Os resultados mostraram que a restauração ativa após 5 anos de implantação apresentou média qualidade do solo, tanto com o cálculo do ISQS com 04 indicadores ou 06 indicadores. Apesar do indicador sintético de qualidade do solo, unir todas as váriaveis estudadas, a observação dos indicadores e suas relações também foi importante. Em nossos resultados foi explícita a variação conjunta dos indicadores de Estrutura do Solo (EST) com os indicadores de DMA e DME, que representam a macro e mesofauna do solo. Também verificamos, forte correlação entre os indicadores de fertilidade do solo e ciclagem de nutrientes, gerando, alta qualidade do solo no Fragmento de Floresta Estacional Semidecidual e média qualidade na Pastagem em uso.

Palavras chaves: Restauração Florestal, Restauração Ativa, Atributos microbiológicos, Mesofauna, Índice de qualidade do solo.

\footnotetext{
ABSTRACT

In forest restoration, it is essential to reestablish the soil's ecological processes, especially in projects that have goals related to climate change mitigation, soil protection and water conservation, in this case, assessing the soil biota can be important. Microbiological attributes of the soil and mesofauna invertebrates that live in the litter, can bring information about the functioning of the soil in different types of use and management of the soil. Converging points between the different soil quality indices consist of choosing the most relevant soil attributes, to integrate them in a single value that varies in a high, medium or low-quality scale. The objective of this work was to integrate the microbiological attributes and diversity of the litter mesofauna as additional indicators in the calculation of the General Indicator of Soil Quality (GISQ, acronym in English), here called Synthetic Indicator of Soil Quality (ISQS), methodology developed by (VELASQUEZ; LAVELLE; ANDRADE, 2007). Using multivariate analyzes, we calculated the indicators: 1) Nutrient cycling (NUT): includes 06 microbiological soil attributes and 2) Mesofauna diversity in the litter (DME): includes density data (ind $\mathrm{m}^{2}$ ) of 18 Taxonomic Groups (GT) of mesofauna in litter and the value of Wealth (number of GT). We evaluated the difference of these indicators in relation to the types of land use: FES (Fragment of Seasonal Semideciduous Forest); RAA (Active Restore A); RAB (Active Restoration B); RAC (Active Restore C); PUC (Pasture for continuous use); PAA (Abandoned Pasture A); PAB (Abandoned Pasture B); PAC (Abandoned Pasture C), in the Atlantic Forest biome, in Itu, São Paulo, Brazil, in the Capoava, Jequitibá and Ingazinho farms. We tested the hypothesis that the value of the synthetic soil quality index (ISQS), in areas in the
} 
process of active restoration is changed when calculated: using four indicators (FER, EST, DMA, AGR) or six indicators (NUT, DME, FER, EST, DMA, AGR). The results showed that the active restoration after 5 years of implantation showed average soil quality, both with the calculation of the ISQS with 04 indicators or 06 indicators. Despite the synthetic indicator of soil quality, uniting all the variables studied, the observation of the indicators and their relationships was also important. In our results, the joint variation of the Soil Structure (EST) indicators with the DMA and DME indicators, which represent the soil macro and mesofauna, was explicit. We also found a strong correlation between soil fertility indicators and nutrient cycling, generating high soil quality in the Seasonal Semideciduous Forest Fragment and medium quality in the Pasture in use.

Key words: Forest Restoration, Active Restoration, Microbiological attributes, Mesofauna, Soil quality index.

\section{INTRODUÇÃO}

Os microrganismos que habitam o solo são os responsáveis, direta ou indiretamente, por processos bioquímicos diversos, que controlam as transformações dos elementos, transferências de energia e nutrientes no sistema solo-planta-atmosfera, constituindo a base de sustentação e produtividade dos ecossistemas terrestes (MOREIRA; SIQUEIRA, 2006). As bactérias e os fungos são propulsores essenciais da mineralização dos nutrientes, participando ativamente dos ciclos do nitrogênio, carbono e fósforo. O comprometimento da atividade microbiana do solo, pode diminuir a síntese e mineralização da matéria orgânica do solo, com consequentes efeitos nos ciclos biogeoquímicos(LAVELLE et al., 2016). Em ecossistemas oligotróficos, as comunidades microbianas do solo podem competir com a vegetação por nutrientes como N e P (CUI; HOLDEN, 2015).

A estrutura da comunidade microbiana varia de acordo com o tipo de uso do solo e possui significante relação, com as variáveis químicas do solo (KANG et al., 2018). Medidas da atividade microbiana no solo podem ser obtidas, pela quantificação da biomassa microbiana, respiração basal, atividade de enzimas e pelo cálculo de alguns quocientes (CARDOSO; VASCONCELLOS, 2015). As enzimas que atuam no solo, não são determinadas por análise direta, mas indiretamente, devido a sua habilidade de transformar um determinado substrato em produtos conhecidos. Indicadores microbianos são mais suscetíveis, às mudanças impostas ao meio ambiente do que indicadores físicos e químicos (CARDOSO et al., 2013).

A análise dos atributos microbiológicos e indicadores bioquímicos podem trazer informações muito relevantes sobre o funcionamento do solo e sua performance na ciclagem e armazenamento dos nutrientes (SANTOS et al., 2011). Em ambientes florestais dados microbiológicos representam grande influencia nos atributos químicofísico do solo e da mesofauna do (ZAGATTO et al., 2019). Invertebrados da mesofauna do solo são pequenos (< $2 \mathrm{~mm}$ ) tais como ácaros, colêmbolos estes invertebrados são influenciados pelas modificações físicas realizadas por organismos da macrofauna (engenheiros do solo) e podem viver em habitats construídos por minhocas, formigas ou cupins, ou seja, invertebrados de menor tamanho corporal encontram recursos alimentares adequados e abrigo dentro das estruturas biogênicas (LAVELLE et al., 2016). Organismos da mesofauna são representados por grupos funcionais do tipo predadores que controlam as populações de outros organismos no solo, e do tipo detritívoros ou decompositores, que contribuem para a decomposição da matéria orgânica e o controle das populações de fungos (MORAES; FRANKLIN, 2008). Um grande número de estudos tem confirmado que as comunidades microbianas são ativadas e selecionadas por engenheiros do ecossistema dentro de suas funções domínios da auto-organização do solo, assim foi construído o conceito de auto organização do solo através de 5 domínios funcionais, estes domínios são como engrenagens que movem o funcionamento do solo (LAVELLE et al., 2016). 
O objetivo deste trabalho foi integrar os atributos microbiológicos e diversidade da mesofauna da serapilheira como indicadores adicionais no cálculo do General Indicator of Soil Quality (GISQ, acrônimo em inglês), aqui denominado Indicador Sintético de Qualidade do Solo (ISQS), metodologia desenvolvida por (VELASQUEZ; LAVELLE; ANDRADE, 2007). Utilizando análises multivariadas calculamos os indicadores: 1)Ciclagem de nutrientes (NUT): com 06 atributos microbiológicos do solo e 2) Diversidade da mesofauna na serapilheira (DME): com dados da densidade (ind $/ \mathrm{m}^{2}$ ) de 18 Grupos Taxonômicos (GT) da mesofauna na serapilheira e o valor da Riqueza $\left(\mathrm{n}^{\circ}\right.$ de GT).

No monitoramento da restauração florestal, são escassos os trabalhos com indicadores edáficos, assim avaliamos o resultados dos diversos indicadores em 8 áreas: FES (Fragmento de Floresta Estacional Semidecidual); RAA (Restauração Ativa A); RAB (Restauração Ativa B); RAC (Restauração Ativa C); PUC (Pastagem de uso contínuo); PAA (Pastagem Abandonada A); PAB (Pastagem Abandonada B); PAC (Pastagem Abandonada C), no bioma Mata Atlântica, em Itu, São Paulo, Brasil. Utilizando o resultados de outros indicadores (FER, EST, DMA e AGR) avaliados nas mesmas áreas, calculamos o ISQS com 6 indicadores. Testamos a hipótese de que o valor do índice sintético de qualidade do solo (ISQS), em áreas em processo de restauração ativa é alterado quando calculado: utilizando quatro indicadores (FER, EST, DMA, AGR) ou seis indicadores (NUT, DME, FER, EST, DMA, AGR).

\section{CONCLUSÃO}

Encontramos grande variação entre os intervalos adequados para cada tipo de indicador, em relação aos tipos de uso analisados. Em cada área estudada, há maior aporte de uma propriedade ou processo, em detrimento de outro, visto que no solo as propriedades e funções são altamente correlacionadas. A baixa qualidade dos indicadores de ciclagem de nutrientes e fertilidade do solo, nas áreas de restauração ativa, é balanceada pela alta qualidade dos indicadores de diversidade da macrofauna do solo e mesofauna da serapilheira, que atuando em sinergia, mantêm a estrutura física e agregação do solo com média e alta qualidade. É importante que a área escolhida como referência alcance os níveis máximos dos parâmetros utilizados, a qualidade edáfica geral no Fragmento de Floresta Atlântica é alta, destacamos os valores altos para os indicadores de ciclagem de nutrientes e fertilidade química do solo podendo indicar que a estabilidade do sistema florestal depende mais destes atributos. Na Pastagem Uso Contínuo apesar de média qualidade da fertilidade do solo e alta qualidade da ciclagem de nutrientes, os indicadores de diversidade (macro e mesofauna) e de estrutura física do solo apresentaram nível baixo de qualidade.

A escolha em monitorar do solo pode ocorrer de acordo com o objetivo do projeto de restauração, por exemplo, onde se almeja melhor produtividade das árvores para fins de exploração econômica sustentável indicadores de qualidade da fertilidade química e da ciclagem de nutrientes devem ser avaliados, em plantios para conter a degradação do solo com em encostas a qualidade da estrutura física deve ser avaliada. Onde o objetivo é a conservação das espécies, os indicadores de diversidade da macrofauna e mesofauna devem ser avaliados.

Uma compreensão mais holística do solo pode ser quantificada no cálculo do Indicador Sintético de Qualidade do Solo (ISQS). O uso de indicadores de qualidade do solo no monitoramento do processo de restauração ecológica pode trazer à tona quais os limiares alcançáveis para o sucesso do restabelecimento dos processos ecológicos e serviços ecossistêmicos do solo, por consequência facilitar a comunicação entre a pesquisa acadêmica e as políticas públicas ambientais. 


\section{REFERÊNCIAS}

AMAZONAS, N. T. et al. Soil macrofauna density and diversity across a chronosequence of tropical forest restoration in southeastern Brazil. Brazilian Journal of Biology, v. 78, n. 3, p. 449-456, 2018.

ANDREWS, S. S.; KARLEN, D. L.; CAMBARDELLA, C. A. The soil management assessment framework: A quantitative soil quality evaluation method. Soil Science Society of America Journal, v. 68, n. 6, p. 1945-1962, 2004. ANZALONE, R. A. et al. Establishing reference values for soil microbial biomass-C in agroecosystems in the Atlantic Forest Biome in Southern Brazil. Ecological Indicators, v. 117, n. March 2019, p. 106586, 2020.

ARONSON, J. What can and should be legalized in ecological restoration? Revista Árvore, v. 34, n. 3, p. 451-454, 2010 .

BENNETT, E. M.; PETERSON, G. D.; GORDON, L. J. Understanding relationships among multiple ecosystem services. Ecology Letters, v. 12, n. 12, p. 1394-1404, 2009.

BRANCALION, P. H. S.; GANDOLFI, S.; RODRIGUES, R. R. Restauração florestal. São Paulo: Oficina de Textos, 2015.

BÜNEMANN, E. K. et al. Soil quality - A critical review. Soil Biology and Biochemistry, v. 120, n. September 2017, p. 105-125, 2018.

CALLAHAM, M. A.; RHOADES, C. C.; HENEGHAN, L. A striking profile: Soil ecological knowledge in restoration management and science. Restoration Ecology, 2008.

CARDOSO, E. J. B. N.; VASCONCELLOS, R. L. F. P. P.-P. Floresta com araucária: composição florística e biota do soloFEALQ, , 2015.

CARDOSO, E. J. N. et al. Soil health : looking for suitable indicators. What should be considered to assess the effects of use and management on soil health ? n. August, p. 274-289, 2013.

CATANI, R. A. Analise quimica para avaliar a fertilidade do solo. Piracicaba: Piracicaba Esalq, 1974.

CHERUBIN, M. R. et al. Soil Quality Indexing Strategies for Evaluating Sugarcane Expansion in Brazil.(Research Article). PLoS ONE, v. 11, n. 3, p. e0150860, 2016.

CUI, J.; HOLDEN, N. M. The relationship between soil microbial activity and microbial biomass, soil structure and grassland management. Soil and Tillage Research, v. 146, n. PA, 2015.

DECAËNS, T. et al. The values of soil animals for conservation biology. European Journal of Soil Biology, v. 42, n. SUPPL. 1, 2006.

DOMINATI, E.; PATTERSON, M.; MACKAY, A. A framework for classifying and quantifying the natural capital and ecosystem services of soils. Ecological Economics, v. 69, n. 9, p. 1858-1868, 2010.

DOMÍNGUEZ-HAYDAR, Y. et al. Evaluation of reclamation success in an open-pit coal mine using integrated soil physical, chemical and biological quality indicators. Ecological Indicators, v. 103, n. March, p. 182-193, 2019.

DORAN, J. W. Soil health and global sustainability: Translating science into practice. Agriculture, Ecosystems and Environment. Anais...fev. 2002Disponível em: <https://linkinghub.elsevier.com/retrieve/pii/S0167880901002468>

EIVAZI, F.; TABATABAI, M. A. Glucosidases and galactosidases in soils. Soil Biology and Biochemistry, v. 20, n. 5, p. 601-606, 1988a.

EIVAZI, F.; TABATABAI, M. A. Glucosidases and galactosidases in soils. Soil Biology and Biochemistry, v. 20, n. 5, p. 601-606, 1 jan. 1988b.

EMMET-BOOTH, J. P. et al. A review of visual soil evaluation techniques for soil structureSoil Use and 
Management, 2016.

FOLGARATI, P. F. Ant biodiversity and its realtionship to ecosystem function: a review. Biodiversity and Conservation, v. 7, p. 1221-1244, 1998.

GATICA-SAAVEDRA, P.; ECHEVERRÍA, C.; NELSON, C. R. Ecological indicators for assessing ecological success of forest restoration : a world review. v. 25, n. 6, p. 850-857, 2017.

GERLACH, J.; SAMWAYS, M.; PRYKE, J. Terrestrial invertebrates as bioindicators: An overview of available taxonomic groups. Journal of Insect Conservation, v. 17, n. 4, p. 831-850, 2013.

GONÇALVES, J. L. DE M. Nutrição e fertilização florestal. Piracicaba: Piracicaba FEALQ, 2000.

GREINER, L. et al. Soil function assessment: review of methods for quantifying the contributions of soils to ecosystem services. Land Use Policy, v. 69, n. September, p. 224-237, 2017.

GUERRA, A. et al. Ecological restoration in Brazilian biomes: Identifying advances and gaps. Forest Ecology and Management, v. 458, n. December, 2020.

HARRIS, J. Soil microbial communities and restoration ecology: Facilitators or followers?Science, 2009.

HENEGHAN, L. et al. Integrating Soil Ecological Knowledge into Restoration Management. Restoration Ecology, v. 16, n. 4, p. 608-617, dez. 2008.

HOLLING, C. S. Understanding the complexity of economic, ecological, and social systems. Ecosystems, v. 4, n. 5, p. 390-405, 2001.

KANG, H. et al. Changes in soil microbial community structure and function after afforestation depend on species and age: Case study in a subtropical alluvial island. Science of the Total Environment, v. 625, p. 1423-1432, 2018.

KARLEN, D. L. et al. Soil quality: humankind's foundation for survival.(Research Editorial)(Editorial). Journal of Soil and Water Conservation, v. 58, n. 4, p. 171, 2003.

LAVELLE, P. et al. Soil invertebrates and ecosystem services. European Journal of Soil Biology, v. 42, n. SUPPL. 1, nov. 2006.

LAVELLE, P. et al. Soil ecosystem services and land use in the rapidly changing orinoco river basin of colombia. Agriculture, Ecosystems and Environment, v. 185, p. 106-117, 2014.

LAVELLE, P. et al. Ecosystem engineers in a self-organized soil: A review of concepts and future research questions. Soil Science, v. 181, n. 3-4, p. 91-109, 2016.

LAVELLE, P. et al. Soil aggregation, ecosystem engineers and the C cycle. Acta Oecologica, v. 105, n. December 2019, p. 103561, 2020.

LONDE, V. et al. Reference and comparison values for ecological indicators in assessing restoration areas in the Atlantic Forest. Ecological Indicators, v. 110, 1 mar. 2020.

MARICHAL, R. et al. Soil macroinvertebrate communities and ecosystem services in deforested landscapes of Amazonia. Applied Soil Ecology, v. 83, p. 177-185, 2014.

MEA, Millennium Ecosystem Assessment series: Ecosystems and Human Well-being: General Synthesis. Island Press, 160pp, 2005.

MENDES, M. S. et al. Look down-there is a gap—the need to include soil data in Atlantic Forest restoration. Restoration Ecology, v. 27, n. 2, p. 361-370, 2019.

MOREIRA, F. M. S. Biodiversidade do solo em ecossistemas brasileiros. Lavras: Lavras UFLA, 2008.

MOREIRA, F. M. S.; SIQUEIRA, J. O. Microbiologia e Bioquímica do Solo. Editora UFLA, v. ed. 2, p. $729,2006$.

NEUTEL, A. M.; HEESTERBEEK, J. A. P.; DE RUITER, P. C. Stability in real food webs: Weak links in long loops. Science, v. 296, n. 5570, p. 1120-1123, 2002. 
NURIA, R. et al. IBQS: A synthetic index of soil quality based on soil macro-invertebrate communities. Soil Biology and Biochemistry, v. 43, n. 10, p. 2032-2045, 2011.

OLIVEIRA, R. O estado da arte da ecologia da restauração e sua relação com a restauração de ecossistemas florestais no bioma Mata Atlântica. Aleph, 2011.

PALACIOS-VARGAS, JOSÉ G, MEJIA RECAMIER B.E., O. A. Guía ilustrada para los artrópodos edáficos. [s.l: s.n.]. v. 20

PEREIRA, N. A. et al. Does the Process of Passive Forest Restoration Affect the Hydrophysical Attributes of the Soil Superficial Horizon? n. 2011, p. 1-15, 2020.

RODRIGUES, R. R. et al. Protocolo de Monitoramento para Programas e Projetos de Restauração FlorestalPacto pela Restauração Florestal da Mata Atlântica, 2013.

RODRIGUES, R. R.; BRANCALION, P. H. S.; NAVE, A. PROGRAMA DE ADEQUAÇÃo AMBIENTAL E RESTAURAÇÃO ECOLÓGICA DAS FAZENDAS CAPOAVA , JEQUITIBÁ E INGAZINHO. Piracicaba: [s.n.].

ROMANELLI, J. P. et al. Assessing ecological restoration as a research topic using bibliometric indicators. Ecological Engineering, v. 120, n. March, p. 311-320, 2018.

ROSSI, M. Mapa pedológico do Estado de São Paulo: revisado e ampliado. São Paulo: [s.n.]. v. 124

RUIZ-JAEN, M. C.; AIDE, T. M. Restoration success: How is it being measured? Restoration Ecology, v. 13, n. 3, p. 569-577, 2005.

SANTOS, D. C. F. et al. Microbial and soil properties in restoration areas in the jequitinhonha valley, Minas Gerais. Revista Brasileira de Ciência do Solo, v. 35, n. 6, p. 2199-2206, 2011.

SCHOENHOLTZ, S. H.; MIEGROET, H. V.; BURGER, J. A. A review of chemical and physical properties as indicators of forest soil quality: challenges and opportunities. Forest Ecology and Management, v. 138, n. 1-3, p. 335-356, 2000.

SNYDER, B. A.; HENDRIX, P. F. Current and potential roles of soil macroinvertebrates (earthworms, millipedes, and isopods) in ecological restoration. Restoration Ecology, v. 16, n. 4, p. 629-636, 2008.

STRASSBURG, B. B. N.; IRIBARREM, A.; BEYER, H. L. Global priority areas for ecosystem restoration. n. August 2019, 2020.

SUGANUMA, M. S.; DURIGAN, G. Indicators of restoration success in riparian tropical forests using multiple reference ecosystems. Restoration Ecology, v. 23, n. 3, p. 238-251, 2015.

TABATABAI, M. A.; BREMNER, J. M. Use of p-nitrophenyl phosphate for assay of soil phosphatase activity. Soil Biology and Biochemistry, v. 1, n. 4, p. 301-307, 1969.

THOUMAZEAU, A. et al. Biofunctool®: a new framework to assess the impact of land management on soil quality. Part A: concept and validation of the set of indicators. Ecological Indicators, v. 97, n. October 2018, p. 100-110, 2019.

VALANI, G. P. et al. Soil quality assessments in integrated crop-livestock-forest systems: A review. Soil Use and Management, n. October, p. 1-15, 2020.

VANCE, E. D.; BROOKES, P. C.; JENKINSON, D. S. An extraction method for measuring soil microbial biomass C. Soil Eiol. Biochem., v. 19, n. 6, p. 1-5, 1987.

VASCONCELLOS, R. L. F. et al. Microbiological indicators of soil quality in a riparian forest recovery gradient. Ecological Engineering, v. 53, p. 313-320, 2013a.

VASCONCELLOS, R. L. F. et al. Soil macrofauna as an indicator of soil quality in an undisturbed riparian forest and 
recovering sites of different ages. European Journal of Soil Biology, v. 58, p. 105-112, 2013b.

VELASQUEZ, E. et al. This ped is my ped: Visual separation and near infrared spectra allow determination of the origins of soil macroaggregates. Pedobiologia, 2007.

VELASQUEZ, E.; LAVELLE, P. Soil macrofauna as an indicator for evaluating soil based ecosystem services in agricultural landscapes. Acta Oecologica, v. 100, n. September, p. 103446, 2019.

VELASQUEZ, E.; LAVELLE, P.; ANDRADE, M. GISQ, a multifunctional indicator of soil quality. Soil Biology and Biochemistry, 2007.

VELOSO, H. P. Classificação da Vegetação Brasileira, Adaptada a um Sistema UniversalRio de Janeiro, 1991.

VIANI, R. A. G. et al. Protocol for monitoring tropical forest restoration: Perspectives from the atlantic forest restoration pact in Brazil. Tropical Conservation Science, v. 10, 2017.

WORTLEY, L.; HERO, J. M.; HOWES, M. Evaluating ecological restoration success: A review of the literature. Restoration Ecology, v. 21, n. 5, p. 537-543, 2013.

ZAGATTO, M. R. G. et al. Interactions between mesofauna, microbiological and chemical soil attributes in pure and intercropped Eucalyptus grandis and Acacia mangium plantations. Forest Ecology and Management, v. 433, n. November 2018, p. 240-247, 2019a.

ZAGATTO, M. R. G. et al. Soil mesofauna in consolidated land use systems: How management affects soil and litter invertebrates. Scientia Agricola, v. 76, n. 2, p. 165-171, 2019 b. 